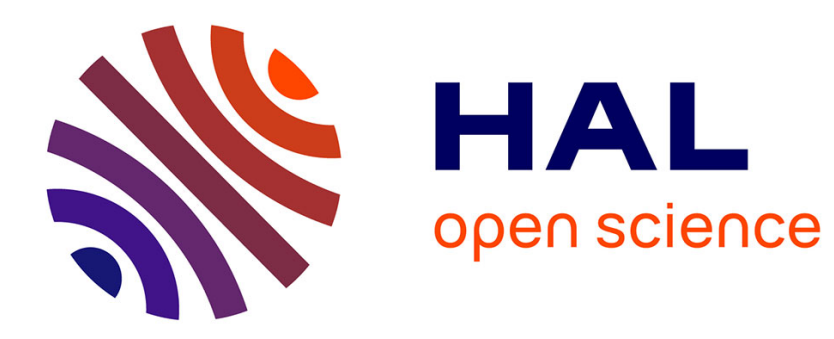

\title{
La démocratie, une quête perpétuelle !
}

\author{
Henri Assogba
}

\section{To cite this version:}

Henri Assogba. La démocratie, une quête perpétuelle !: Réponse à Danielle Béatrice Ongono Bikoe et à Amadou Moctar Diallo. Éthique publique - revue internationale d'éthique sociétale et gouvernementale , 2011, Dialogues pour réinventer la démocratie, 13 (2), 10.4000/ethiquepublique.788 . hal-01782013

\section{HAL Id: hal-01782013 \\ https://hal.science/hal-01782013}

Submitted on 1 May 2018

HAL is a multi-disciplinary open access archive for the deposit and dissemination of scientific research documents, whether they are published or not. The documents may come from teaching and research institutions in France or abroad, or from public or private research centers.
L'archive ouverte pluridisciplinaire HAL, est destinée au dépôt et à la diffusion de documents scientifiques de niveau recherche, publiés ou non, émanant des établissements d'enseignement et de recherche français ou étrangers, des laboratoires publics ou privés. 
Revue internationale d'éthique sociétale et gouvernementale

vol. $13, \mathrm{n}^{\circ} 2 \mid 2011$

Dialogues pour réinventer la démocratie

\section{La démocratie, une quête perpétuelle !}

Réponse à Danielle Béatrice Ongono Bikoe et à Amadou Moctar Diallo

\section{Henri Assogba}

\section{(2) OpenEdition}

Journals

\section{Édition électronique}

URL : http://journals.openedition.org/ethiquepublique/788

DOI : $10.4000 /$ ethiquepublique.788

ISSN : 1929-7017

Éditeur

Éditions Nota bene

\section{Édition imprimée}

Date de publication : 31 décembre 2011

ISBN : 978-2-89518-402-7

ISSN : 1488-0946

Ce document vous est offert par Bibliothèque de l'Université Laval

\section{s. \\ LAVAL}

Bibliothèque

Référence électronique

Henri Assogba, «La démocratie, une quête perpétuelle! », Éthique publique [En ligne], vol. 13, n

2 | 2011, mis en ligne le 23 octobre 2012, consulté le 29 mars 2018. URL : http://

journals.openedition.org/ethiquepublique/788; DOI : 10.4000/ethiquepublique.788

Ce document a été généré automatiquement le 29 mars 2018

Tous droits réservés 


\title{
La démocratie, une quête perpétuelle!
}

\author{
Réponse à Danielle Béatrice Ongono Bikoe et à Amadou Moctar Diallo
}

\author{
Henri Assogba
}

1 Mardi 4 octobre 2011, il est $8 \mathrm{~h} 40$ à Québec. Il y a une dizaine de minutes environ que je suis devant le nouveau document vierge ouvert sur mon écran d'ordinateur, me demandant par où commencer ma réponse aux deux pertinentes réflexions sur la démocratie en Afrique subsaharienne signées par Danielle Béatrice Ongono Bikoe (Cameroun) et Amadou Moctar Diallo (Sénégal). Également connecté à Internet, j'écoute en direct, d'une oreille distraite, le journal « Afrique-midi » (décalage horaire oblige) de Radio France International. C'est ainsi que j'apprends que l'élection présidentielle au Cameroun aura lieu le dimanche suivant (9 octobre 2011) avec 23 candidats en lice. Ce n'est pas tant, hélas, l'issue du scrutin qui m'interpelle dans cette nouvelle. Mais ce qui retient le plus mon attention (et m'offre un point d'ancrage pour cet échange), c'est la vox populi diffusée dans ce bulletin d'information radiophonique avec cette jeune fille qui se désole de n'avoir connu depuis sa naissance que le même homme à la présidence de son pays ; Paul Biya, pour ne pas le nommer, est encore candidat à sa propre succession. Puis, la demoiselle de poursuivre: « [...] j'en ai assez de ce simulacre de démocratie [...]. » Répondre aux deux belles plumes sélectionnées dans le cadre du concours « Notre démo cratie! » est bel et bien me situer au cœur de l'actualité.

2 Mais avant d'aller plus loin dans cet échange, je tiens à faire quelques petites clarifications. Même si le continent africain demeure celui que je connais le moins mal à ce jour pour y avoir vécu et travaillé en tant que journaliste, cela ne m'autorise guère à revendiquer une expertise absolue dans les expériences démocratiques qui s'y mènent. Mieux, et ce n'est pas un scoop, il n'y a pas une Afrique mais des Afriques. D'autres voix, plus autorisées que la mienne, existent et en ont fait leur terrain de recherche. Je ne réponds donc pas ès qualité et que le lecteur n'attende pas de ma part des propos universitaires ou ex cathedra, encore moins des recettes miracle universelles sur la démocratie. J'assume donc entièrement la subjectivité de mes propos qui demeurent ceux d'un citoyen ordinaire dans une agora. 


\section{Des idées très pertinentes}

3 Cela dit, revenons à cette jeune Camerounaise désabusée citée plus haut dont le diagnostic semble proche de celui de sa compatriote Danielle Béatrice Ongono Bikoe qui écrit que " les Africains ne croient pas en une démocratie en Afrique ". Je peux comprendre et je comprends le désarroi d'une génération de Camerounais à l'égard de sa classe politique, mais je ne serai pas aussi péremptoire dans ma sentence et je ne me permettrai pas de l'étendre à toute l'Afrique. Et puis, comme il est mentionné dans la présentation du concours, si les raisons diffèrent d'un continent à un autre, voire d'un pays à un autre (Bayart, 2009 ; Guèye, 2009; Banégas, 2003 ; Mbembe, 2000 ; etc.), la crise de la démocratie représentative libérale n'est pas une particularité africaine. Face à ce constat qui semble général, ce sont donc les propositions concrètes qui méritent qu'on s'y attarde.

Pour sa part, Danielle Béatrice Ongono Bikoe évoque pour l'Afrique des actions à mener aussi bien en dehors du processus électoral que pendant les élections. Sa proposition pour une éducation à la citoyenneté (qu'elle soit officielle ou non) comme une action prioritaire me convient. En effet, dans plusieurs pays africains en cours de démocratisation, il arrive que des populations longtemps privées de liberté et de parole passent d'un extrême à un autre en confondant la démocratie et l'anarchie. Et c'est à juste titre que Danielle Béatrice Ongono Bikoe rappelle que « [...] la politique est une bataille d'idéologies et qu'elle ne devrait pas dégénérer en conflits armés [...] ». Mieux informées et mieux éduquées, les populations se laisseront moins manipuler par certains acteurs politiques qui n'ont pas ou peu d'idées, d'arguments et de programmes à faire valoir.

Quant à la proposition visant à établir la crédibilité des institutions démocratiques par une séparation des pouvoirs, de telles dispositions existent déjà, du moins dans les textes fondamentaux. La question est de savoir comment rendre "véritable » cette séparation des pouvoirs. On peut essayer de revoir les clés de répartition et les règles de nomination ou de cooptation, mais on ne peut jamais être à l'abri des " retournements de veste ", des alliances, contre-alliances et bien d'autres combinaisons imprévisibles.

En ce qui concerne les actions relatives aux élections proprement dites, Danielle Béatrice Ongono Bikoe recommande de renoncer au multipartisme intégral et avance même que le nombre idéal de clivages ou de tendances politiques devrait être de cinq. Par principe, je me méfie de tout ce qui doit se mouler dans un cadre rigide et restrictif, d'autant plus que les intentions qui ont servi, par exemple, à justifier la création des partis uniques ou partis-États dans les pays africains nouvellement indépendants étaient bonnes : l'unité au service du développement de ces pays. Des intentions vite dévoyées et qui ont conduit à des régimes antidémocratiques. C'est peut-être naïf de ma part, mais j'ai la faiblesse de croire en une sorte de " sélection naturelle » qui va finir par s'opérer dans ces États postcoloniaux plus récents qui s'inscrivent dans un processus de démocratisation. C'est vrai qu'avec 23 candidats pour la présidentielle camerounaise, on peut se poser des questions. Au Bénin aussi, des questions similaires ont agité le landernau politique et la réponse provisoire a été d'augmenter de façon substantielle la caution à payer pour tenter de décourager les « candidatures farfelues ».

7 L'organisation des élections libres et transparentes est la dernière action que préconise Danielle Béatrice Ongono Bikoe avec à la clé une simplification et une fiabilité plus grande des procédures d'inscription des électeurs. 
8 Même si les régimes démocratiques sont souvent jugés à l'aune des élections, l'originalité de la contribution proposée par Amadou Moctar Diallo est de montrer que le fait de réduire la démocratie à l'organisation d'élections revient à vider celle-ci de tout son sens. Son invitation à aller au-delà des élections me semble être une bonne approche, surtout dans les pays nouvellement engagés sur la voie démocratique. C'est d'autant plus vrai quand Amadou Moctar Diallo écrit que « pour être vitale, la démocratie doit se sentir quotidiennement dans la participation effective des citoyens ». Dans la quête de cette vitalité démocratique, je note, dans les deux contributions des lauréats, que l'information et la formation du citoyen sont indispensables. Et c'est à juste titre qu'Amadou Moctar Diallo se demande « quel sens donner au vote si le citoyen n'est pas conscient de l'enjeu de son acte ».

Partant du substrat de ces propositions pertinentes, je nourris la réflexion en y ajoutant trois axes principaux complémentaires qui, de mon point de vue, contribuent à une revitalisation de la démocratie en Afrique subsaharienne.

\section{De la nécessité de mener des réformes structurelles profondes}

10 La démocratie ne doit pas être perçue comme une incantation, une recette miracle ou la panacée censée guérir tous les maux qui minent le continent africain. À mon humble avis, on ne peut pas réfléchir à l'avenir de la démocratie ex nihilo en faisant abstraction des contextes. Une approche holistique me semble nécessaire. Comment rendre fiable, par exemple, un fichier électoral (sujet emblématique au cœur de nombreuses contestations) en l'absence d'un système d'état civil adéquat? On peut, dans l'urgence, tenter de contourner la difficulté en se faisant livrer à grands frais des kits biométriques. Mais en dehors des élections, une profonde réforme du système de l'état civil doit être menée et poursuivie. De l'avis de plusieurs observateurs, de nombreux dirigeants politiques africains plus ou moins surpris par le début de la vague de démocratisation semblent désormais utiliser des artefacts démocratiques surtout destinés aux bailleurs de fonds qui en ont fait une conditionnalité de leur aide. Liberté de la presse? Démonopolisation du secteur audiovisuel? Liberté d'opinion? Liberté d'association? Qu'à cela ne tienne, un cadre institutionnel et législatif est mis en place mais dans les faits, les mauvaises " vieilles bonnes habitudes » se perpétuent avec, par exemple, d'importants contrats de promotion signés avec des groupes de presse privés qui sont, dans certains pays, jugés « pires " qu'au temps des médias d'État. L'organisation plus ou moins chaotique et périodique des élections participe de cette stratégie. C'est pourquoi, sans remettre en cause le bien-fondé des élections et tout en travaillant à améliorer leur tenue régulière, je suis d'avis avec Amadou Moctar Diallo qu'il faut aller au-delà des élections et se soucier de la démocratie au quotidien. Je pense qu'il faut rendre endogène le processus de démocratisation pour mieux l'enraciner et éviter une démocratisation d'affichage tournée vers l'extérieur. Toutefois, il faut garder à l'esprit, quel que soit le degré de maturité du système politique d'un pays, la célèbre sentence de lord Acton : « Le pouvoir corrompt ; le pouvoir absolu corrompt absolument » (lord Acton, 1988). Jeune journaliste en formation au Sénégal (Centre d'études des sciences et techniques de l'information), j'ai couvert avec une grande excitation en 2000 la campagne électorale et les premières confé rences de l'icône de l'opposition $\mathrm{M}^{\mathrm{e}}$ Abdoulaye Wade, nouvellement élu dans sa maison du Point E. Deux mandats présidentiels après, celui qui se présentait comme le candidat du sopi (c'est-à-dire le changement) et de la jeunesse veut rempiler à nouveau et propose de 
modifier les règles constitutionnelles. De pareils exemples sont légion. Et c'est là où j'en viens au deuxième axe de ma proposition.

\section{Prôner et adopter une culture de l'éthique publique}

11 Que le lecteur ne se méprenne pas sur mes intentions en voyant dans cette seconde proposition une approche moralisante, voire de « donneur de leçons ». Telle n'est pas ma vocation, mais je n'hésite pas, à la suite de Paul Ricœur (1985: 67), à donner une signification éthique non pas seulement à la prudence demandée aux gouvernants, mais encore à l'engagement du citoyen dans une démocratie. D'ailleurs, j'estime que la démocratie gagnerait beaucoup à réhabiliter, par exemple, le sens des valeurs, le respect de la parole donnée, la transparence dans la gestion des biens publics, le sens d'un vote. La formation d'une opinion publique libre dans son expression et la mise en place d'une veille citoyenne sont des actions que je préconise.

\section{S'inscrire dans un processus d'amélioration}

12 La démocratie, comme on le voit, ne s'octroie pas, mais elle s'acquiert difficilement et se construit progressivement en fonction des réalités liées au contexte. Elle est une quête, voire une conquête permanente. C'est un système politique toujours perfectible. Il peut arriver qu'il y ait des remises en cause. Mais sa force, c'est de s'inspirer de ses échecs. Au propre comme au figuré, la démocratie a un coût et toute la question est de savoir si nous sommes prêts à payer ce coût.

Post-scriptum : Laissez-moi profiter encore - ça ne va pas durer éternellement - de la facilité de prédiction des résultats électoraux dans certains pays d'Afrique subsaharienne et prédire dès maintenant où je termine cette réponse le nom du gagnant de l'élection présidentielle du Cameroun : Paul Biya, au pouvoir depuis 1982...

\section{BIBLIOGRAPHIE}

ACTON, John Emerich Edward Dalberg, et J. Rufus FEARs (1988), Selected Writings of Lord Acton :

Essays in Religion, Politics and Morality, Indianapolis, Liberty Fund Inc.

BANÉGAS, Richard (2003), La démocratie à pas de caméléon : transition et imaginaires politiques, Paris, Éditions Karthala.

BAYART, Jean-François (2009), «La démocratie à l'épreuve de la tradition en Afrique subsaharienne», Pouvoirs, nº 129 (avril), p. 27-44.

GUÈYE, Babacar (2009), «La démocratie en Afrique : succès et résistances», Pouvoirs, nº 129 (avril), p. 5-26.

MBEMBE, Achille (2000), «Entre coups d'Etat, élections reportées et mouvements sociaux : esquisses d'une démocratie à l'africaine», Le Monde Diplomatique, n 559, p. 20-21. 
RICCUR, Paul (1985), «Ethique et politique», Autres Temps, n 5, p. 58-70.

\section{RÉSUMÉS}

Éduquer à la citoyenneté, limiter le nombre de formations politiques, simplifier et rendre plus crédible l'organisation des élections, élargir la réflexion au-delà des élections pour mieux appréhender la vitalité démocratique d'un pays, voilà quelques-unes des actions concrètes proposées par deux jeunes Africains pour revitaliser la démocratie. J'y ajoute des pistes complémentaires comme la nécessité de mener des réformes structurelles profondes, la promotion et l'adoption d'une culture de l'éthique publique et enfin l'inscription dans un processus d'amélioration continue.

In this article, two young Africans propose concrete steps to revitalize democracy : for example, offering citizenship education, limiting the number of political parties, streamlining the organization of elections and making it more credible, and broadening reflection beyond elections so as to better assess countries' democratic vitality. I propose some additional steps such as making in-depth structural reforms, promoting and adopting a culture of public ethics, and deploying a process of ongoing improvement.

\section{INDEX}

Keywords : citizens, democracy, election, public ethics, subsaharian Africa

Mots-clés : Afrique subsaharienne, citoyens, démocratie, élections, éthique publique

\section{AUTEUR}

\section{HENRI ASSOGBA}

Ancien journaliste et titulaire d'un doctorat en sciences de l'information et de la communication, Henri Assogba est actuellement professeur adjoint au Département d'information et de communication de l'Université Laval, à Québec. Ses recherches portent sur les mutations du journalisme avec un intérêt marqué pour la médiatisation des thématiques environnementales. 